\title{
The Effect of Vibratory Stimulation on the Timed-Up-and-Go Mobility Test: A Pilot Study for Sensory-Related Fall Risk Assessment
}

\author{
Nima TOOSIZADEH ${ }^{1,2,3}$, Genevieve WAHLERT ${ }^{1}$, Mindy FAIN ${ }^{2,3}$, Jane MOHLER $^{1}$ \\ ${ }^{1}$ Department of Biomedical Engineering, University of Arizona, Tucson, Arizona, USA, ${ }^{2}$ Division \\ of Geriatrics, General Internal Medicine and Palliative Medicine, Department of Medicine, \\ University of Arizona, USA, ${ }^{3}$ Arizona Center on Aging, Department of Medicine, University of \\ Arizona, USA
}

Received February 14, 2020

Accepted April 29, 2020

Epub Ahead of Print July 16, 2020

\section{Summary}

Effects of localized lower-extremity vibration on postural balance have been reported. The purpose of the current study was to investigate the effect of low-frequency vibration of calf muscles on the instrumented Timed-Up-and-Go (iTUG) test among older adults. Older adults were recruited and classified to low $(n=10$, age $=72.9 \pm 2.8$ years $)$ and high fall risk $(n=10$, age $=83.6 \pm 9.6)$ using STEADI. Vibratory system $(30 \mathrm{~Hz}$ or $40 \mathrm{~Hz})$, was positioned on calves along with wearable motion sensors. Participants performed the iTUG test three times, under conditions of no-vibration, $30 \mathrm{~Hz}$, and $40 \mathrm{~Hz}$ vibration. Percentage differences in duration of iTUG components were calculated comparing vibration vs no-vibration conditions. Significant between-group differences were observed in iTUG $(p=0.03)$; high fall risk participants showed reduction in the duration of turning $(-10 \%$ with $30 \mathrm{~Hz} ; p=0.15$ and $-15 \%$ with $40 \mathrm{~Hz} ; p=0.03$ ) and turning and sitting $(-18 \%$ with $30 \mathrm{~Hz} ; p=0.02$ and $-10 \%$ with $40 \mathrm{~Hz}$; $p=0.08)$. However, vibration increased turning ( $+18 \%$ with $30 \mathrm{~Hz} ; p=0.20$ and $+27 \%$ with $40 \mathrm{~Hz} ; p=0.12$ ) and turning and sitting duration ( $+27 \%$ with $30 \mathrm{~Hz} ; p=0.11$ and $+47 \%$ with $40 \mathrm{~Hz} ; p=0.12$ ) in low fall risk participants. Findings suggest that lower-extremity vibration affects dynamic balance; however, the level of this influence may differ between low and high fall risk older adults, which can potentially be used for assessing agingrelated sensory deficits.

\section{Key words}

Older Adults - Wearable sensors - Proprioceptive • Dynamic balance $\bullet$ Vibration stimulation $\bullet$ Fall risk

\section{Corresponding author}

Nima Toosizadeh, Arizona Center on Aging (ACOA), Department of Biomedical Engineering, University of Arizona, College of Engineering, Bioscience Research Lab (BSRL), 1601 E Helen St., Tucson AZ, 85719, United States. Email: ntoosizadeh@arizona.edu

\section{Introduction}

Aging precipitates many physiological and functional changes in the human body, which result in impaired function and mobility. As we age, the ability to execute the complex skill of maintaining body equilibrium declines due to a variety of factors. Sarcopenia, the loss of muscle mass, and dynapenia, the loss of muscle power, are common consequences of aging-related muscle degeneration (Clark and Manini 2010, Manini and Clark 2011, Yeung et al. 2019). Additional loss of innervating neural muscle fibers from demyelination can result in decreased signal transmission velocity, which affects the ability to quickly respond to axonal stimulation in muscle activation (Goble et al. 2009). These conditions, coupled with further degeneration of proprioceptors within muscle spindles and tendon organs can diminish muscle length and force sensation, resulting in unsteady and inconsistent body motion (Horak and Nashner 1986, Inglis et al. 1994). These physiological changes culminate in an increase of postural instability, and ultimately can lead to loss of balance and falls. Falls, in addition to being alarmingly prevalent, can be notably detrimental to older adults, 
leading to injury, reduction in quality of life and independence, and even death. Approximately $30 \%$ of adults aged 65 and older experience one or more falls each year, potentially resulting in injury, hospitalization or fatality (Liu-Ambrose et al. 2015, Mohler et al. 2016). The Timed Up and Go (TUG) test is a validated approach to assess mobility and fall risk among older adults, which is widely used and recommended by the American and British Geriatric Societies for assessment of fall risk (Panel on Prevention of Falls in Older Persons and Society 2011). TUG is a composite measure of functional mobility, which incorporates multiple neuromuscular components, and the ability to sit, stand, turn, and walk is predictive of future disability and fall (Bohannon, 2006, Podsiadlo and Richardson 1991, Shumway-Cook et al. 2000). Traditionally, the TUG test involved the sole measure of the whole testing duration; however, more recently, the new sensor-based instrumented TUG (iTUG) method provides the opportunity to extract spatio-temporal parameters during each of the sitting, standing, turning, and walking components (Toosizadeh et al. 2015, Zampieri et al. 2010).

In continuation of research in fall risk assessment based on mobility measures, the purpose of the current study was to implement lower-extremity vibratory stimulation to magnify sensorimotor deficits in performing iTUG. Among healthy individuals, vibratory stimulation adversely influences the range and speed of body sway during upright standing (Čapičikova et al. 2006, Caudron et al. 2010, Duclos et al. 2014, Ehsani et al. 2018a, Patel et al. 2009, Radhakrishnan et al. 2011, Toosizadeh et al. 2018a). We previously investigated the effects of mechanical calf vibration on postural balance among healthy and high fall risk older adults (Ehsani et al. 2018, Toosizadeh et al. 2018a, Toosizadeh et al. $2018 \mathrm{~b})$. Within low frequency vibration $(30-40 \mathrm{~Hz})$ among a small sample of 20 older adults, we observed significant differences in balance behaviors due to vibration among the groups (Ehsani et al. 2018, Toosizadeh et al. 2018a). Within the eyes-closed condition, high fall risk participants showed $70 \%$ less vibration-induced changes in medial-lateral body sway (due to less ankle sway), and $54 \%$ less sway velocity, when compared to healthy elderly participants $(p<0.001$; effect size $=0.6-1.4$ ). This observation suggests a reduced proprioceptive performance among high fall risk elders, which led to less alteration in postural sway due to muscle vibration. Interestingly, within our pilot project we observed that more than half of high fall risk individuals (likely those with sensory deficits) showed improvements in balance (reduced overall COG sway) when exposed to vibration, while less than $10 \%$ in the low fall risk group showed improvements (Ehsani et al. 2018, Toosizadeh et al. 2018a). Accordingly, we hypothesize that vibratory stimulation would influence dynamic balance as well; however, the level of this influence depends on the level of aging-related sensory impairments. To test this hypothesis iTUG was performed with and without calf vibration among low and high fall risk older adults, to explore how lower-extremity stimulation would influence routine daily activities, including sitting, standing, walking, and turning.

\section{Methods}

\section{Participants}

Two groups of participants were recruited, including 10 low fall risk (age $=73 \pm 3$ years) and 10 high fall risk (age $=84 \pm 9$ years) older adults aged 65 and older. High fall risk participants were selected according to the Center for Disease Control and Prevention's STEADI Risk for Falling Assessment (Rubenstein et al. 2011), which involves four questions, assigning one point to each affirmative response: 1) Have you fallen in the past year? 2) Are you worried about falling? 3) Do you feel unsteady when you are walking? and 4) Have you had two or more falls? Those with a score of zero or one without a history of falling were considered low fall risk, and those with a score of two to four were considered high fall risk. Exclusion criteria for both groups were: disorders associated with severe motor deficits and balance performance, including stroke, Parkinson's disease, dementia (Mini Mental State Examination (MMSE) score <20) (Folstein et al. 1975), severe arthritis in lower-extremities, cancer or diabetic neuropathy, vestibular diseases, lower-extremity ulceration and amputation, history of dizziness, vertigo, and sedating medication or alcohol consumption within the prior 24 hours. The above mentioned disorders were identified using subjective questionnaires as defined in previous work (Speechley and Tinetti 1991, Tinetti and Speechley 1989), and participants were excluded if they claimed to have any related symptoms. For the low fall risk group, an additional exclusion criterion of fall incident in a prior year was considered. All participants were recruited after completing written informed consent according to the 
principles expressed in the Declaration of Helsinki (World 2009), approved by the Review Board of the University of Arizona.

\section{Clinical measurements}

Prior to testing, participants filled out clinical questionnaires, including: 1) the visual analog pain scale for lower-extremity (VAS-10) (0: no pain; 10: extreme pain) (Langley and Sheppeard, 1985) within the prior 2-week period and at the time of the visit; 2) short falls efficacy scale international (Short FES-I) for assessing the fear of falling (Kempen et al. 2007); 3) the fourquestion fall scale (see above); and 4) the number of falls (defined as 0,1 , or 2 or more within a prior year). The fear of falling and lower-extremity pain were assessed here since they are both associated with fall risk among older adults (Murphy et al. 2003, Tomita et al. 2015).

\section{iTUG assessments}

Each participant performed four iTUG tests, including: one practice trial with no vibration system attached, one trial with vibration system on calves but with no stimulation, one trial with $30 \mathrm{~Hz}$ vibration, and one trial with $40 \mathrm{~Hz}$ vibration. Of note, data from practice trials were not used in the analysis. Each trial comprised of the participant rising from a seated position (STS1), walking to a predetermined position three meters away (W1), turning (T1), walking back to the chair (W2), and turning and sitting down (T2\&STS2). Angular acceleration was estimated using two wearable motion sensors each equipped with a tri-axial gyroscope (LEGSys, BioSensics, Boston, MA, USA), which were attached to shins on both sides. Using sensor data, the timing of each of the above iTUG components (STS1, W1, T1, W2, and T2\&STS2) were identified. Since the turning and sitting tasks are not distinct, overlapping in the turning and sitting task was estimated as the duration of the T2\&STS2 over the sum of separate T2 and STS2 duration, representing the sitting strategy (Weiss et al. 2019). The percentage change in the duration of task completion was estimated comparing stimulation conditions (30 Hz and $40 \mathrm{~Hz}$ vibration) versus the no-stimulation condition (baseline).

\section{Vibration stimulation}

Mechanical vibration of 30 and $40 \mathrm{~Hz}$ frequencies and $1 \pm 0.002 \mathrm{~mm}$ amplitude were imposed to both gastrocnemius muscles continuously, using custom- made eccentric rotating servomotor. Velcro straps were used to attach the vibrators to the belly of each muscle. Based on previous studies to assure that effects of stimulation reach a plateau level, participants were exposed to a one-minute warm-up vibration prior to each test (Čapičikova et al. 2006, Tjernström et al. 2002). Each warm-up vibration exposure occurred before starting the iTUG test while the participant was sitting on the chair. To minimize the residual effects of vibration on iTUG performance (Čapičikova et al. 2006, Wierzbicka et al. 1998), participants had a minimum of two-minute rest period between trials. The vibration turned off immediately after the participant finished the iTUG test. Further, to minimize the residual effects of vibration, instead of randomizing the trials, tests with no vibration was performed first, followed by 30 or $40 \mathrm{~Hz}$ stimulation trials. Of note, the order of 30 or $40 \mathrm{~Hz}$ vibrations was randomized. This study design was implemented because following a practice session, the learning effect due to repeating the iTUG test is negligible. Therefore, it is expected that the differences in iTUG performance between vibration and no-vibration trials would mainly represent vibration-induced alterations with minimum residual effects.

\section{Statistical analysis}

Differences in demographic parameters among low and high fall risk participants were assessed using one-way analysis of variance (ANOVA) models. Differences in subjective questionnaires, as well as the baseline no-vibration iTUG parameters were assessed using multivariable ANOVA models, considering fall risk groups (low versus high), age, gender, and body mass index (BMI) as independent variables. To assess differences in vibration-induced iTUG changes between fall risk groups, multivariable repeated measures ANOVA models were used. In each model, percentage change in balance parameters due to vibration (compared to the baseline condition with no stimulation) were considered as dependent variables; fall risk groups, age, gender, BMI, and vibration frequency (within subject variable) were considered as independent variables. Cohen's effect size was calculated for each ANOVA test. The interaction effect between fall risk groups and vibration frequency was also assessed. Matched-paired $t$-test was used to assess significant changes in iTUG performance within each of the low and high fall risk group. Further analyses were performed to assess the 
association between baseline no-vibration iTUG and vibration-induced iTUG performance, using Pearson correlations (r). Lastly, correlations between subjective questionnaires (i.e., the pain score, FES-I, and the fall score) and vibration-induce changes in balance parameters were assessed using linear regression models and reported as Pearson correlations. All analyses were done using JMP (Version 14, SAS Institute Inc., Cary, $\mathrm{NC}$, USA), and statistical significance was concluded when $\mathrm{p}<0.05$.

\section{Results}

\section{Participants}

Between low and high fall risk groups, age, FES-I, and fall score were significantly different $(p<0.01$, Table 1). All other demographic and clinical measures were not significantly different between groups $(p>0.07$, Table 1).

Table 1: Sociodemographic Information and clinical measures for low and high fall risk participants. Significant between fall risk group differences are indicated with asterisks.

\begin{tabular}{|c|c|c|c|}
\hline Variables & Low Fall Risk & High Fall Risk & $p$-value \\
\hline Number, $n$ ( $\%$ of total) & $10(50 \%)$ & $10(50 \%)$ & - \\
\hline Female, $n$ ( $\%$ of group) & $6(60 \%)$ & $7(70 \%)$ & 0.99 \\
\hline Age, year $(S D)$ & $72.90(2.81)$ & $83.60(9.46)$ & $0.01 *$ \\
\hline Stature, cm (SD) & $165.03(10.91)$ & $165.62(11.21)$ & 0.91 \\
\hline Body mass, $k g(S D)$ & $64.71(8.37)$ & $65.24(16.39)$ & 0.93 \\
\hline$B M I, k g / m^{2}(S D)$ & $23.75(2.11)$ & $23.52(4.08)$ & 0.87 \\
\hline Pain at the moment, 0-10 (SD) & $0.20(0.63)$ & $1.90(2.69)$ & 0.07 \\
\hline Pain within two weeks, 0-10 (SD) & $0.80(2.53)$ & $3.50(3.72)$ & 0.07 \\
\hline Short FES-I, 7-28 (SD) & $8.00(1.63)$ & $14.90(3.96)$ & $<0.001^{*}$ \\
\hline Fall score, 0-4 (SD) & $0.10(0.32)$ & $3.10(0.74)$ & $<0.001 *$ \\
\hline Number of falls within one year (SD) & $0.00(0)$ & $0.80(0.92)$ & $<0.01 *$ \\
\hline
\end{tabular}
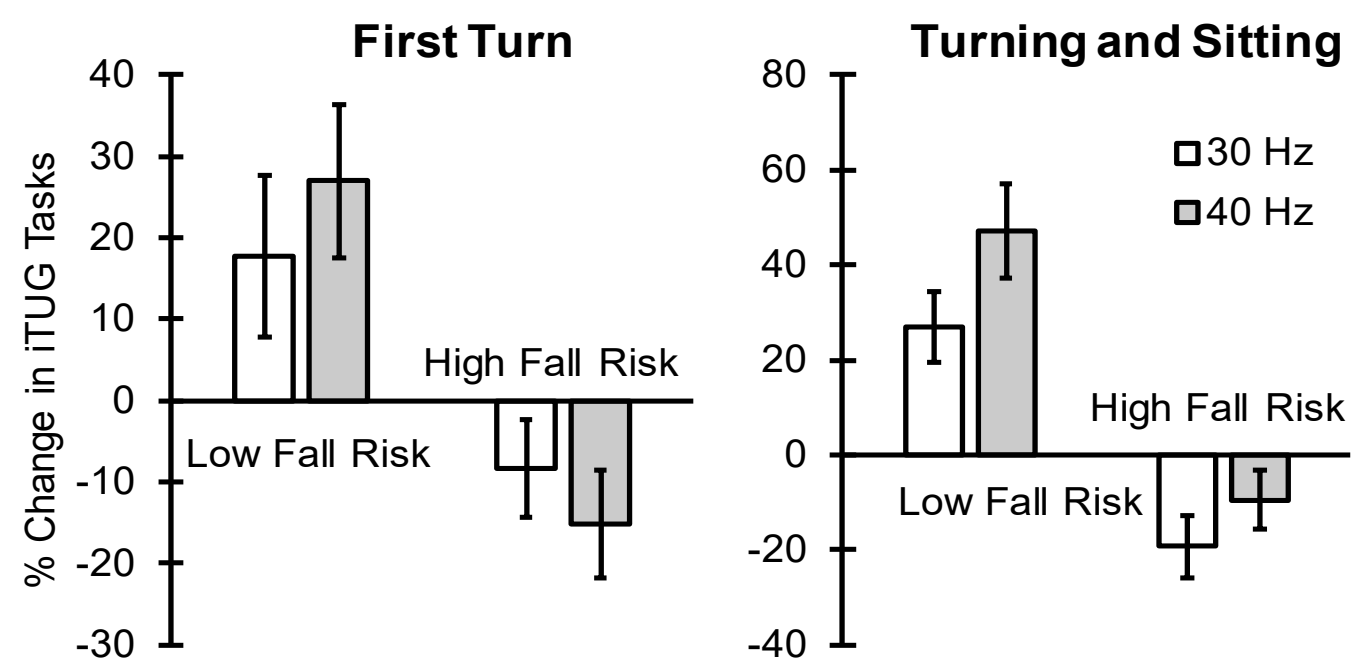

Fig. 1. Changes in instrumented Timed-Up-and-Go (iTUG) performance comparing vibration versus no-vibration trials.

\section{iTUG and vibration}

Although the normal baseline iTUG test showed significant univariate between-group differences in the task completion duration (e.g., total iTUG duration of
$11.26 \pm 2.75 \mathrm{~s}$ for low-fall risk compared to $23.97 \pm 10.00 \mathrm{~s}$ for high-fall risk, $p<0.01$ ), none of these differences were significant when the model was adjusted with age, gender, and BMI $(p>0.12)$. After the vibration was 
applied, iTUG performance altered among both the low and high fall risk groups. Overall, low fall risk participants performed the iTUG test slower after vibration, while, the performance improved among high fall risk individuals. For the high fall risk group, applied vibration resulted in a $10 \pm 19 \%(p=0.15)$ and $15 \pm 21 \%$ $(p=0.03)$ faster completion of the T1 task, as well as a $18 \pm 27 \%(p=0.02)$ and $10 \pm 20 \%(p=0.08)$ improvement for the combined task of T2\&STS2 during $30 \mathrm{~Hz}$ and $40 \mathrm{~Hz}$ trials, respectively (Table 2 and Fig. 1). On the other hand, low fall risk participants were observed to have declined performance, which was presented as $18 \pm 31 \% \quad(p=0.20)$ and $27 \pm 61 \%(p=0.12)$ increase in completion time for $\mathrm{T} 1$, and $27 \pm 46 \% \quad(p=0.11)$ and $47 \pm 95 \%(p=0.12)$ increase for T2\&STS2 completion during $30 \mathrm{~Hz}$ and $40 \mathrm{~Hz}$ trials, respectively (Table 2 and Fig. 1). Accordingly, ANOVA models showed significant differences between low and high fall risk groups in task completion duration changes for $\mathrm{T} 1$ and $\mathrm{T} 2+\mathrm{STS} 2$ $(p=0.03)$, when adjusted for age, gender, and BMI. Although not significant $(p=0.09)$, this trend was also observed for the overall iTUG duration. No significant between-group and within-group differences were observed for sitting strategies $(p>0.19)$. Further, no significant interaction effect between fall risk and vibration frequency was found $(p>0.20)$.

Independent of the fall risk group classification, current results showed negative associations between baseline iTUG performance and alterations in iTUG performance when participants exposed to the vibratory stimulation. As illustrated in Fig. 2, changes in T1 and T2\&STS2 duration were significantly (and negatively) correlated with the initial baseline duration for completing these tasks ( $r=0.51-0.77$ and $p<0.03$ for both $30 \mathrm{~Hz}$ and $40 \mathrm{~Hz}$ trials). Further, significant negative correlations were observed between $\mathrm{T} 1$ and T2\&STS2 changes within $30 \mathrm{~Hz}$ vibration with the fall score $(r=0.50-0.55, p<0.03)$. Although similar negative trends were noticeable for other correlations between subjective questionnaires and iTUG performance, none of them were significant $(p>0.17)$.

Table 2.

\begin{tabular}{|c|c|c|c|c|}
\hline iTUG Task & Low Fall Risk & High Fall Risk & $p$-value GROUP & Effect Size \\
\hline \multicolumn{5}{|c|}{ Sit to Stand (STS1) } \\
\hline $30 H Z(S D)$ & $0.66(1.14)$ & $0.08(0.62)$ & \multirow{2}{*}{0.19} & \multirow{2}{*}{0.36} \\
\hline $40 H Z(S D)$ & $0.58(0.82)$ & $0.05(0.52)$ & & \\
\hline \multicolumn{5}{|c|}{ Walk three meters (W1) } \\
\hline $30 \mathrm{HZ}(S D)$ & $-0.09(0.23)$ & $0.06(0.21)$ & \multirow[b]{2}{*}{0.85} & \multirow[b]{2}{*}{0.26} \\
\hline $40 H Z(S D)$ & $-0.04(0.33)$ & $0.05(0.20)$ & & \\
\hline \multicolumn{5}{|c|}{ Turn around (T1) } \\
\hline $30 H Z(S D)$ & $0.18(0.31)$ & $-0.10(0.19)$ & \multirow{2}{*}{$0.03 *$} & \multirow{2}{*}{0.53} \\
\hline $40 H Z(S D)$ & $0.27(0.61)$ & $-0.15(0.21)$ & & \\
\hline \multicolumn{5}{|c|}{ Walk back to chair (W2) } \\
\hline $30 H Z(S D)$ & $-0.01(0.37)$ & $0.11(0.37)$ & \multirow{2}{*}{0.81} & \multirow{2}{*}{0.33} \\
\hline $40 H Z(S D)$ & $-0.12(0.27)$ & $0.11(0.18)$ & & \\
\hline \multicolumn{5}{|c|}{ Turn and sit down (T2\&STS2) } \\
\hline $30 H Z(S D)$ & $0.27(0.46)$ & $-0.13(0.27)$ & \multirow{2}{*}{$0.03 *$} & \multirow{2}{*}{0.51} \\
\hline $40 H Z(S D)$ & $0.47(0.95)$ & $-0.10(0.20)$ & & \\
\hline \multicolumn{5}{|c|}{ Total duration } \\
\hline $30 H Z(S D)$ & $0.11(0.26)$ & $-0.03(0.23)$ & \multirow{2}{*}{0.09} & \multirow{2}{*}{0.29} \\
\hline $40 H Z(S D)$ & $0.14(0.32)$ & $-0.02(0.17)$ & & \\
\hline
\end{tabular}

Percent change in the duration of completion of iTUG tasks after applying vibratory stimulation. Results from age, gender, and BMI adjusted repeated measure ANOVA models for between group differences are presented. Significant between fall risk group differences are indicated with asterisks. 

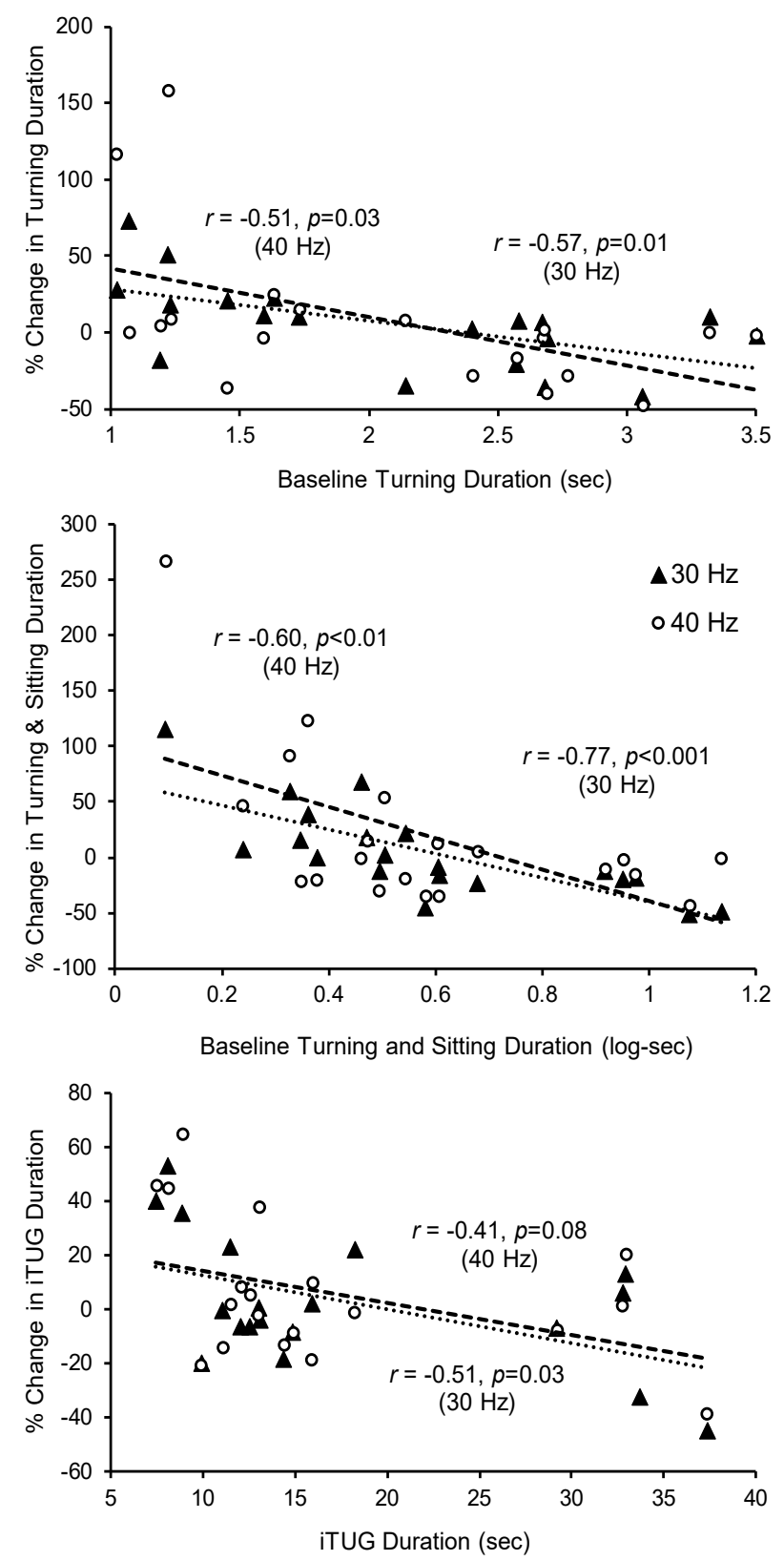

Fig. 2. Correlations between changes in instrumented Timed-Upand-Go (iTUG) performance due to vibration and baseline iTUG performance.

\section{Discussion}

\section{Effects of vibration on $i T U G$}

In agreement with the current theoretical hypothesis and our previous findings for postural balance (Ehsani et al. 2018, Toosizadeh et al. 2018a, Toosizadeh et al. 2018b), we observed that the effect of vibratory stimulation differed significantly among low and high fall risk older adults. Out of 10 low fall risk older adults within the current sample, eight showed an overall deterioration in iTUG performance when repeating the task with the vibration. On the other hand, nine out of ten high fall risk elders showed improved iTUG performance with vibration compared to baseline, which was mainly represented by shorter durations of turning and sitting. Accordingly, within the current sample, adding the vibration to iTUG noticeably improved the identification of fall risk compared to the common iTUG test, as iTUG performance was not significantly different between the two groups. Within the current vibration-based iTUG approach we aimed to reduce some between-subject differences in physical activity performance, by calculating the percentage changes in iTUG performance for each subject when exposed to outside vibration. In the other word, the overall iTUG performance was normalized using the baseline performance, with the purpose of solely focusing on proprioceptive differences between the fall risk groups. Therefore, components of the iTUG test that were expected to more sensitively alter with proprioceptive stimulation (i.e., turning and sitting), showed a significant between-group vibration-induced changes.

Turning and changing direction tends to be a challenging motor task for older adults, especially those influenced by Parkinson's disease, low back pain, as well as those who are prone to falling (Hulbert et al. 2015, Toosizadeh et al. 2016, Yamada et al. 2012). Qualitative analyses of turning mechanism have suggested differences in turning strategies due to aging-related motor function deficits, which was observed by a tendency for performing spin turns (i.e., ipsilateral turns: left turn while the left limb is the stance limb) among older adults compared to step turns (i.e., contralateral turns: left turn while the right limb is the stance limb) among young individuals (Akram et al. 2010, Fino et al. 2015). Further, case-control research among older adults demonstrated that falling while turning had the highest likelihood of hip fracture with an odds ratio of about eight (compared to an odds ratio of one for normal walking) (Cumming and Klineberg 1994). Although evidence exist that turning is a demanding task in terms of neuromuscular burden, to the best of our knowledge, no study exists to assess the association between a disturbed turning task and fall risk. Current findings suggest that an efficient execution of the turning task may highly depend on proprioceptive performance. Hypothetically, low fall risk older adults with more intact proprioceptive performance showed a compromised turning performance when exposed to vibration, while high fall risk elders benefitted from vibration to improve 
the turning task execution. Of course, this hypothesis requires further investigation with more accurate assessment of proprioceptive performance before testing.

Performing a turning task becomes even more challenging when it is followed by sitting, which requires an accurate proprioceptive sensation to provide efficient timing of muscle activation to safely lower the body center of mass (Parvaneh et al. 2017, Weiss et al. 2019). Previous work suggested different sitting strategies among older adults, including distinct-strategy (cautious sitting) and overlapping-strategy (Parvaneh et al. 2017, Weiss et al. 2019). Within the distinct-strategy, turning and walking would be fully completed and then the task of sitting takes place; while, within the overlappingstrategy individuals tend to perform turning/walking and sitting concurrently. Results from these previous studies suggested that frail elders and those with Parkinson's disease tend to implement a cautious sitting strategy, which involves a more prolonged time delay between turning/walking and sitting (Parvaneh et al. 2017, Weiss et al. 2019). Although current results showed vibrationinduced improvement and deterioration in turning and sitting performance among high and low fall risk participants, respectively, our further analysis showed no difference in the sitting strategies; the amount of overlapping before and after vibration was not significantly different between either groups $(p>0.19)$.

\section{Effect of vibration on proprioceptive performance - Theoretical explanation}

It was hypothesized that the observed different responses to the vibratory stimulation within the iTUG test among fall risk groups may be attributed to the differences in age-related sensory performance. These influences were explained by the fact that mechanical vibration of muscle can increase excitement of type Ia afferents in spindles, and increase the excitability of muscle motor neurons (Burke et al. 1976, Wierzbicka et al. 1998). Signals from muscle spindles are directed to motor neurons, which activate the parent muscles to restore joint position (i.e., the ankle joint) within a shortlatency reflexive mechanism (Horak and Nashner, 1986). Thus, lower-extremity muscle vibration can affect this reflexive mechanism by altering the interaction between sensory spindles and the muscle motor executive system. Also, proprioceptive feedback from muscle spindles provides information regarding the level of motor activities, which is processed in the brain cortex to adjust muscle activity (Hulliger, 1984, Mihara et al. 2008).
Muscle vibration can cause some illusionary sensation in the brain regarding the lower-extremity position, and consequently influences the long-latency responses (Goble et al. 2009, Roll et al. 1989). With aging, the efficiency of the reflexive loop declines due to changes in covering capsule dimensions, reduced number of intrafusal fibers within spindles, and denervation process (Goble et al. 2009). Also aging of the central nervous system can cause reduction in attentional resources and a general loss of neural substrate (Raz and Rodrigue, 2006). Although these two hypothetical mechanisms can explain the observed between-group differences in response to vibration, the concept of mechanical stimulation effects on proprioceptive performance needs to be validated within future studies. More specifically, it would be critical to understand how mechanical vibration can influence the ankle joint position sense, kinesthesia, force sense, and more importantly the postural balance feedback mechanism.

\section{Limitations and future direction}

Due to some limitations, findings from the current study should be interpreted cautiously, and further confirmations are required. One of these limitations was the small sample size. Although results are promising, the number of participants within each group was limited and may not be representative of a wide range of agingrelated neuromuscular deficits that can possibly lead to the observed between-group differences. Also, high fall risk participants were selected based on the history of fall and poor balance. Therefore, no direction conclusion can be made regarding the association between aging-related lower-extremity proprioceptive deficits and vibration effects. Our findings, however, showed that regardless of fall risk categories, elders with worse iTUG performance benefited more from the vibratory stimulation.

Other limitation of the current study was to have a few testing conditions for the vibration exposures. Findings cannot inform what vibration areas (gastrocnemius vs. peroneus longus) or vibration frequencies (lower frequency $(30 \mathrm{~Hz}-40 \mathrm{~Hz})$ vs. higher frequency $\sim 80 \mathrm{~Hz}$ ) could have greater effects on iTUG. Of note, the vibration area and frequency were selected based on previous studies on postural balance (Abrahámová et al. 2009, Ehsani et al. 2018, Ivanenko et al. 1999, Toosizadeh et al. 2018a). This limitation needs to be addressed within future systematic studies of vibration effects on both postural balance as well as dynamic balance. 
Lastly, a two-minute rest period was allocated between trials, the vibration frequencies were randomized, and the no-vibration trial was designed to be performed before any exposure to calf vibration; however, some confounding vibration residual effects may still exist. To overcome this limitation, in larger studies, sessions should be done in separate days to completely eliminate the residual effects of vibration on iTUG performance.

\section{Conclusions}

Within our sample of high fall risk older adults, we observed that vibration improved the performance of more demanding components of the iTUG test including turning and sitting. Interestingly, the effect of vibration was adverse among low fall risk participants. Accordingly, current findings suggest that adding vibratory stimulation to the gastrocnemius muscle can be used to assess dynamic balance performance within the iTUG test. We believe that the main effect of vibration is on muscle spindles, which can in turn influence the proprioceptive performance of lower-extremities. The concept of vibratory stimulation for assessing proprioceptive performance has high potential to inform clinical screening and future applications in fall risk prevention. Current promising findings, although preliminary, may lay the groundwork to promote lowerextremity vibratory stimulation for improving postural and dynamic balance among elders at high fall risk.

\section{Conflict of Interest}

There is no conflict of interest.

\section{Acknowledgements}

We thank Marilyn Gilbert for clinical coordination. We thank Ashley Scott, Yun Mei, and Richard Huang for data collection.

\section{References}

ABRAHÁMOVÁ D, MANCINI M, HLAVAČKA F, CHIARI L: The age-related changes of trunk responses to Achilles tendon vibration. Neurosci Lett 467: 220-224, 2009. https://doi.org/10.1016/j.neulet.2009.10.041

AKRAM SB, FRANK JS, CHENOURI S: Turning behavior in healthy older adults: is there a preference for step versus spin turns? Gait Posture 31: 23-26, 2010. https://doi.org/10.1016/j.gaitpost.2009.08.238

BOHANNON RW: Reference values for the timed up and go test: a descriptive meta-analysis. Journal of geriatric physical therapy 29: 64-68, 2006. https://doi.org/10.1519/00139143-200608000-00004

BURKE D, HAGBARTH K-E, LÖFSTEDT L, WALLIN BG: The responses of human muscle spindle endings to vibration of non-contracting muscles. J Physiol 261: 673-693, 1976. https://doi.org/10.1113/jphysiol.1976.sp011580

ČAPIČIKOVA N, ROCCHI L, HLAVAČKA F, CHIARI L, CAPELLO A: Human postural response to lower leg muscle vibration of different duration. Physiol Res 55 (Suppl 1): S129-S134, 2006.

CAUDRON S, NOUGIER V, GUERRAZ M: Postural challenge and adaptation to vibration-induced disturbances. Exp Brain Res 202: 935-941, 2010. https://doi.org/10.1007/s00221-010-2194-6

CLARK BC, MANINI TM: Functional consequences of sarcopenia and dynapenia in the elderly. Curr Opin Clin Nutr Metab Care 13: 271, 2010. https://doi.org/10.1097/MCO.0b013e328337819e

CUMMING RG, KLINEBERG RJ: Fall frequency and characteristics and the risk of hip fractures. J Am Geriatr Soc 42: 774-778, 1994. https://doi.org/10.1111/j.1532-5415.1994.tb06540.x

DUCLOS NC, MAYNARD L, BARTHELEMY J, MESURE S: Postural stabilization during bilateral and unilateral vibration of ankle muscles in the sagittal and frontal planes. J Neuroeng Rehabil 11: 130, 2014. https://doi.org/10.1186/1743-0003-11-130

EHSANI H, MOHLER J, MARLINSKI V, RASHEDI E, TOOSIZADEH N: The influence of mechanical vibration on local and central balance control. J Biomech 71: 59-66, 2018. https://doi.org/10.1016/j.jbiomech.2018.01.027

FINO P, FRAMES C, LOCKHART T: Classifying step and spin turns using wireless gyroscopes and implications for fall risk assessments. Sensors 15: 10676-10685, 2015. https://doi.org/10.3390/s150510676

FOLSTEIN MF, FOLSTEIN SE, MCHUGH PR: "Mini-mental state": a practical method for grading the cognitive state of patients for the clinician. J Psychiatr Res 12: 189-198, 1975. https://doi.org/10.1016/0022-3956(75)90026-6 
GOBLE DJ, COXON JP, WENDEROTH N, VAN IMPE A, SWINNEN SP: Proprioceptive sensibility in the elderly: degeneration, functional consequences and plastic-adaptive processes. Neurosc Biobeh Rev 33: 271-278, 2009. https://doi.org/10.1016/j.neubiorev.2008.08.012

HORAK FB, NASHNER LM: Central programming of postural movements: adaptation to altered support-surface configurations. J Neurophysiol 55: 1369-1381, 1986. https://doi.org/10.1152/jn.1986.55.6.1369

HULBERT S, ASHBURN A, ROBERT L and VERHEYDEN G: A narrative review of turning deficits in people with Parkinson's disease. Disabil Rehabil 37: 1382-1389, 2015. https://doi.org/10.3109/09638288.2014.961661

HULLIGER M: The mammalian muscle spindle and its central control. In: Reviews of Physiology, Biochemistry and Pharmacology. Rev Physiol Biochem Pharmacol, Volume 101. Springer, 1984, pp. 1-110. https://doi.org/10.1007/BFb0027694

INGLIS JT, HORAK FB, SHUPERT CL, JONES-RYCEWICZ C: The importance of somatosensory information in triggering and scaling automatic postural responses in humans. Exp Brain Res 101: 159-164, 1994. https://doi.org/10.1007/BF00243226

IVANENKO YP, TALIS VL, KAZENNIKOV OV: Support stability influences postural responses to muscle vibration in humans. Eur J Neuros 11: 647-654, 1999. https://doi.org/10.1046/j.1460-9568.1999.00471.x

KEMPEN GI, YARDLEY L, VAN HAASTREGT JC, ZIJLSTRA GR, BEYER N, HAUER K, TODD C: The Short FES-I: a shortened version of the falls efficacy scale-international to assess fear of falling. Age Ageing 37: 45-50, 2007. https://doi.org/10.1093/ageing/afm157

LANGLEY G, SHEPPEARD H: The visual analogue scale: its use in pain measurement. Rheumatol Int 5: 145-148, 1985. https://doi.org/10.1007/BF00541514

LIU-AMBROSE T, DAVIS JC, HSU CL, GOMEZ C, VERTES K, MARRA C, BRASHER PM, DAO E, KHAN KM, COOK W: Action Seniors! -secondary falls prevention in community-dwelling senior fallers: study protocol for a randomized controlled trial. Trials 16: 144, 2015. https://doi.org/10.1186/s13063-015-0648-7

MANINI TM, CLARK BC: Dynapenia and aging: an update. J Gerontol A Biol Sci Med Sci 67: 28-40, 2011. https://doi.org/10.1093/gerona/glr010

MIHARA M, MIYAI I, HATAKENAKA M, KUBOTA K, SAKODA S: Role of the prefrontal cortex in human balance control. Neuroimage 43: 329-336, 2008. https://doi.org/10.1016/j.neuroimage.2008.07.029

MOHLER MJ, WENDEL CS, TAYLOR-PILIAE RE, TOOSIZADEH N, NAJAFI B: Motor performance and physical activity as predictors of prospective falls in community-dwelling older adults by frailty level: application of wearable technology. Gerontology 62: 654-664, 2016. https://doi.org/10.1159/000445889

MURPHY SL, DUBIN JA, GILL TM: The development of fear of falling among community-living older women: predisposing factors and subsequent fall events. J Gerontol A Biol Sci Med Sci 58: M943-M947, 2003. https://doi.org/10.1093/gerona/58.10.M943

PANEL ON PREVENTION OF FALLS IN OLDER PERSONS AGS, SOCIETY BG: Summary of the updated American Geriatrics Society/British Geriatrics Society clinical practice guideline for prevention of falls in older persons. J Am Geriatr Soc 59: 148-157, 2011. https://doi.org/10.1111/j.1532-5415.2010.03234.x

PARVANEH S, MOHLER J, TOOSIZADEH N, GREWAL GS, NAJAFI B: Postural transitions during activities of daily living could identify frailty status: application of wearable technology to identify frailty during unsupervised condition. Gerontology 63: 479-487, 2017. https://doi.org/10.1159/000460292

PATEL M, MAGNUSSON M, KRISTINSDOTTIR E, FRANSSON P-A: The contribution of mechanoreceptive sensation on stability and adaptation in the young and elderly. Eur J Appl Physiol 105: 167-173, 2009. https://doi.org/10.1007/s00421-008-0886-4

PODSIADLO D, RICHARDSON S: The timed "Up \& Go": a test of basic functional mobility for frail elderly persons. J Am Geriatr Soc 39: 142-148, 1991. https://doi.org/10.1111/j.1532-5415.1991.tb01616.x

RADHAKRISHNAN SM, HATZITAKI V, PATIKAS D, AMIRIDIS IG: Responses to Achilles tendon vibration during self-paced, visually and auditory-guided periodic sway. Exp Brain Res 213: 423, 2011. https://doi.org/10.1007/s00221-011-2792-y

RAZ N, RODRIGUE KM: Differential aging of the brain: patterns, cognitive correlates and modifiers. Neurosc Biobeh Rev 30: 730-748, 2006. https://doi.org/10.1016/j.neubiorev.2006.07.001 
ROLL J, VEDEL J, RIBOT E: Alteration of proprioceptive messages induced by tendon vibration in man: a microneurographic study. Exp Brain Res 76: 213-222, 1989. https://doi.org/10.1007/BF00253639

RUBENSTEIN LZ, VIVRETTE R, HARKER JO, STEVENS JA, KRAMER BJ: Validating an evidence-based, selfrated fall risk questionnaire (FRQ) for older adults. J Safety Res 42: 493-499, 2011. https://doi.org/10.1016/j.jsr.2011.08.006

SHUMWAY-COOK A, BRAUER S, WOOLLACOTT M: Predicting the probability for falls in community-dwelling older adults using the Timed Up \& Go Test. Phys Ther 80: 896-903, 2000. https://doi.org/10.1093/ptj/80.9.896

SPEECHLEY M, TINETTI M: Falls and injuries in frail and vigorous community elderly persons. J Am Geriatr Soc 39: 46-52, 1991. https://doi.org/10.1111/j.1532-5415.1991.tb05905.x

TINETTI ME, SPEECHLEY M: Prevention of falls among the elderly. N Engl J Med 320: 1055-1059, 1989. https://doi.org/10.1056/NEJM198904203201606

TJERNSTRÖM F, FRANSSON P-A, HAFSTRÖM A, MAGNUSSON M: Adaptation of postural control to perturbations-a process that initiates long-term motor memory. Gait Posture 15: 75-82, 2002. https://doi.org/10.1016/S0966-6362(01)00175-8

TOMITA Y, ARIMA K, KANAGAE M, OKABE T, MIZUKAMI S, NISHIMURA T, ABE Y, GOTO H, HORIGUCHI I, AOYAGI K: Association of physical performance and pain with fear of falling among community-dwelling Japanese women aged 65 years and older. Medicine 94: 2015. https://doi.org/10.1097/MD.0000000000001449

TOOSIZADEH N, EHSANI H, MIRAMONTE M, MOHLER J: Proprioceptive impairments in high fall risk older adults: the effect of mechanical calf vibration on postural balance. Biomed Eng Online 17: 51, $2018 \mathrm{a}$. https://doi.org/10.1186/s12938-018-0482-8

TOOSIZADEH N, HARATI H, YEN T-C, FASTJE C, MOHLER J, NAJAFI B, DOHM M: Paravertebral spinal injection for the treatment of patients with degenerative facet osteoarthropathy: Evidence of motor performance improvements based on objective assessments. Clin Biomech 39: 100-108, 2016. https://doi.org/10.1016/j.clinbiomech.2016.10.007

TOOSIZADEH N, MOHLER J, LEI H, PARVANEH S, SHERMAN S, NAJAFI B: Motor performance assessment in Parkinson's disease: association between objective in-clinic, objective in-home, and subjective/semi-objective measures. PloS one 10: e0124763, 2015. https://doi.org/10.1371/journal.pone. 0124763

TOOSIZADEH N, MOHLER J, MARLINSKI V: Low intensity vibration of ankle muscles improves balance in elderly persons at high risk of falling. PLoS one 13: e0194720, 2018b. https://doi.org/10.1371/journal.pone.0194720

WEISS A, HERMAN T, MIRELMAN A, SHIRATZKY SS, GILADI N, BARNES LL, BENNETT DA, BUCHMAN AS, HAUSDORFF JM: The transition between turning and sitting in patients with Parkinson's disease: A wearable device detects an unexpected sequence of events. Gait Posture 67: 224-229, 2019. https://doi.org/10.1016/j.gaitpost.2018.10.018

WIERZBICKA M, GILHODES J, ROLL J: Vibration-induced postural posteffects. J Neurophysiol 79: 143-150, 1998. https://doi.org/10.1152/jn.1998.79.1.143

WORLD MAI: Declaration of Helsinki. Ethical principles for medical research involving human subjects. J Indian Med Assoc 107: 403, 2009.

YAMADA M, HIGUCHI T, MORI S, UEMURA K, NAGAI K, AOYAMA T, ICHIHASHI N: Maladaptive turning and gaze behavior induces impaired stepping on multiple footfall targets during gait in older individuals who are at high risk of falling. Arch Gerontol Geriatr 54: e102-e108, 2012. https://doi.org/10.1016/j.archger.2011.08.012

YEUNG SS, REIJNIERSE EM, PHAM VK, TRAPPENBURG MC, LIM WK, MESKERS CG, MAIER AB: Sarcopenia and its association with falls and fractures in older adults: A systematic review and meta-analysis. J Cachexia Sarcopenia Muscle 2019. https://doi.org/10.1002/jcsm.12411

ZAMPIERI C, SALARIAN A, CARLSON-KUHTA P, AMINIAN K, NUTT JG, HORAK FB: The instrumented timed up and go test: potential outcome measure for disease modifying therapies in Parkinson's disease. J Neurol Neurosurg 81: 171-176, 2010. https://doi.org/10.1136/jnnp.2009.173740 